\title{
Assessing language dominance with functional MRI: The role of control tasks and statistical analysis
}

\author{
Frank Dodoo-Schittko $^{\mathrm{a}, *}$, Katharina Rosengarth ${ }^{\mathrm{a}}$, Christian Doenitz ${ }^{\mathrm{b}}$, Mark W. Greenlee ${ }^{\mathrm{a}}$ \\ a Institute for Experimental Psychology, University of Regensburg, Regensburg, Germany \\ ${ }^{\mathrm{b}}$ Institute of Neurosurgery, University Medical Center of Regensburg, Germany
}

\section{A R T I C L E I N F O}

\section{Article history:}

Received 21 October 2011

Received in revised form

20 July 2012

Accepted 21 July 2012

Available online 27 July 2012

\section{Keywords:}

Lateralization

Broca's

Presurgical

Semantic

Generation

\begin{abstract}
A B S T R A C T
There is a discrepancy between the brain regions revealed by functional neuroimaging techniques and those brain regions where a loss of function, either by lesion or by electrocortical stimulation, induces language disorders. To differentiate between essential and non-essential language-related processes, we investigated the effects of linguistic control tasks and different analysis methods for functional MRI data. Twelve subjects solved two linguistic generation tasks: (1) a verb generation task and (2) an antonym generation task (each with a linguistic control task on the phonological level) as well as two decision tasks of semantic congruency (each with a cognitive high-level control task). Differential contrasts and conjunction analyses were carried out on the single-subject level and an individual lateralization index ( $\mathrm{LI}$ ) was computed. On the group level we determined the percent signal change in the left inferior frontal gyrus (IFG: BA 44 and BA 45). The conjunction analysis of multiple language tasks led to significantly greater absolute LIs than the LIs based on the single task versus fixation contrasts. A further significant increase of the magnitude of the LIs could be achieved by using the phonological control conditions. Although the decision tasks appear to be more robust to changes in the statistical threshold, the combined generation tasks had an advantage over the decision tasks both for assessing language dominance and locating Broca's area. These results underline the need for conjunction analysis based on several language tasks to suppress highly task-specific processes. They also point to the need for high-level cognitive control tasks to partial out general, language supporting but not language critical processes. Higher absolute LIs, which reflect unambiguously hemispheric language dominance, can be thus obtained.
\end{abstract}

(c) 2012 Elsevier Ltd. All rights reserved.

\section{Introduction}

A longstanding challenge in the preoperative diagnosis of patients who will undergo brain surgery is to localize the neuronal correlates of higher cognitive functions involved in language and memory. Damage to critical cortical areas is associated with paresis, aphasia or amnesia. Since these disorders impact the quality of life, they need to be taken into account when conducting a risk-benefit analysis for brain surgery. The neural correlates of phonology, semantics, morphology and syntax are normally located in the left language-dominant hemisphere. Approximately $10 \%$ of healthy persons show an inverse pattern with right-sided language dominance (Pujol, Deus, Losilla, \& Capdevila, 1999), predominantly in left-handed persons (Knecht et al., 2000; Loring et al., 1990;

\footnotetext{
* Correspondence to: Universitaet Regensburg, Institute for Experimental Psychology, Universitaetsstrasse 31, 93053 Regensburg, Germany. Tel.: + 49941943 3249; fax: +49941943 3233 .

E-mail address: frank.dodoo-schittko@psycholgie.uni-regensburg.de (F. Dodoo-Schittko)
}

Rasmussen \& Milner 1977). In studies using the Wada test (Wada \& Rasmussen, 1960) mixed language dominance is often found with evidence for the involvement of both hemispheres (Risse, Gates, \& Fangmana, 1997). Rasmussen and Milner (1977) reported that 7\% of the right handed patients suffering from epilepsy showed language disturbance after anesthetizing both the left and the right supply area of the arteria cerebri media. Even though Rasmussen and Milner (1977) found no brain damage in patients with mixed language dominance these patients had epilepsy, indicating pathological brain function or micro-lesions that went undetected with the radiological techniques at that time. Such micro-lesions could lead to plasticity and reorganization of brain networks, suggesting that the percentage of healthy persons with mixed language dominance is lower than that reported in Rasmussen and Milner (1977) study. In addition mixed language dominance in terms of a diffuse allocation of speech areas over both hemispheres seems to be linked to infantile brain lesions that trigger massive neuronal reorganization processes, or to psychiatric disorders like autism (Kleinhans, Müller, Cohenc, \& Courchesnee, 2008; Whitehouse and Bishop (2008)), dyslexia (Baillieux et al., 2009; Milne, Syngeniotis, 
Jackson, \& Corballis, 2002) or schizophrenia (Bleich-Cohen, Hendler, Kotler, \& Strous, 2009; Li et al., 2007; Sommer, Ramsey, Kahn, Aleman, \& Bouma, 2001). For healthy persons hemispheric language dominance usually expresses itself in an unambiguous left or right fashion (Knecht et al., 2003; Ojemann, Ojemann, Lettich, \& Berger, 1989; Schäffler, Lüders, \& Beck, 1996). These observations contrast to the results of brain imaging studies of language dominance in healthy persons (Ramsey, Sommer, Rutten, \& Kahn, 2001; Szaflarski, Holland, Schmithorst, \& Byars, 2006; van Ettinger-Veenstra et al., 2010). The lateralization indices (LIs) of these studies often cannot be reliably used to classify persons as left or right language dominant. It is well established that the right hemisphere contributes to language functions (Lindell, 2006). However, impairments in linguistic processes following lesions of the non-dominant hemisphere often go unnoticed in daily life. As a consequence aphasiologists have primarily focused on the dominant (left) hemisphere. For clinical applications robust estimates of cerebral lateralization of speech function in daily life would be useful.

Conducting a meta-analysis on 129 scientific reports using functional neuroimaging techniques, Vigneau et al. (2006) revealed 730 activation peaks for phonological, semantic and syntactic processing scattered over most of the left hemisphere. Intraoperative electrocortical stimulation (IES) mapping of tumor or epilepsy patients reveals that only a small fraction of these regions is critical for language (FitzGerald et al., 1997; Kho et al., 2005). This mismatch between regions revealed by functional neuroimaging techniques and those revealed by lesion or by IES suggests that brain imaging uncovers not only language critical regions but also non-essential regions that support the language network. Such non-essential language functions are, on the one hand, cognitive processes like working memory, attention or perception (Bookheimer et al., 1997; FitzGerald et al., 1997; Ramsey et al., 2001). Using a "combined task analysis" of different language tasks, which were examined with conjunction analysis, Ramsey et al. (2001) reported an overlap between brain activation evoked by single linguistic tasks. As a result, highly task-specific processes go unheeded and only activation remain that reflect fundamental language processes. Furthermore the appropriate selection of control conditions is important to determine language dominance and to map language areas with brain imaging. Simple fixation of gaze on a central point, passive rest or a task with low cognitive demands are often used as control conditions (Engström, Ragnehed, Lundberg, \& Söderfeldt, 2004). In this context Binder et al. (1999) emphasized that a passive rest condition leads to higher activation contrasted to an auditory perception condition in the left temporoparietal network. Similar activations to the passive rest condition were reported for a linguistic semantic condition. This indicates that the control conditions are important with respect to the detection of hemispheric language dominance based on fMRI results. Binder (2011) showed this for nonlinguistic control tasks. Based on activation from tasks with different linguistic complexity differential contrasts can be computed that reveal the neuronal correlates of the cognitive process of interest (Newman \& Twieg, 2001). The control of sensory input, attention, grapheme-phoneme conversion, phonological processing and articulation (Riecker et al., 2000), which plays an important role even in covert speech tasks (Wildgruber, Ackermann, \& Grodd, 2001), can help to discriminate between semantic from non-semantic domains (Wildgruber et al., 2001). In this way, purely semantic processes, assumed to be strongly lateralized and reflective of general hemispheric language dominance, should be differentiated from other cognitive processes.

The control of task performance represents a further demand. Paradigms often include semantic word generation (Brannen et al., 2001; Rutten, Ramsey, van Rijen, Alpherts, \& van Veelen, 2002a;
Xiong et al., 2000), verbal fluency (Amunts et al., 2004; Gaillard et al., 2000) or picture naming tasks (Hunter et al., 1999; Rutten et al., 2002a; Rutten, Ramsey, van Rijen, Noordmans, \& van Veelen, 2002b) where the participants are usually instructed to produce covert speech to avoid motion artifacts or activation of primary motor areas. Performed in this way no behavioral data can be recorded. A problem in the clinical setting is whether the patients have actually generated words within the defined time interval in covert verbal fluency paradigms (Adcock, Wise, Oxbury, Oxbury, \& Mattews, 2003, Jansen et al., 2006; Hertz-Pannier et al., 2002). Thus we created semantic congruency decision tasks along with semantic generation tasks to obtain behavioral data and to compare the ability of these tasks to assess hemispheric language dominance and to localize eloquent brain areas.

\section{Material and method}

\subsection{Subjects}

Twelve healthy (three male, mean age $=27(\mathrm{SD}=5.2)$ ) volunteers without any history of neurologic or psychiatric disorders participated in this study. The test of handedness (Edinburgh Inventory (Oldfield, 1971)) revealed that 11 subjects were completely right-handed with no left-handed first- or second-degree relatives. One subject was left-handed with a left-handed mother. Because of the greater probability of right-sided language dominance, this subject was excluded from the analysis on group level of the functional data. All participants spoke German as their native language.

\subsection{Experimental setup and material}

This study included four different paradigms each consisting of an experimental and a control condition. The order of the paradigms was randomized across subjects. The stimuli were presented visually in written form on a projection screen. A mirror fixed at the head coil reflected the participant's view towards the screen. The first two paradigms concerned language generation tasks (a verb generation task (VG)) (Fiez, Raichle, Balota, Tallal, \& Petersen, 1996; Ramsey et al., 2001, Rutten et al., 2002a, 2002b; Xiong et al., 2000) and an antonym generation task (AG) (Ramsey et al., 2001; Rutten et al., 2002a, 2002b). In case of verb generation subjects had to generate an appropriate verb that semantically matched a noun (e.g., car-drive) whereas the antonym generation task required the generation of an antonym according to a presented adjective (e.g., "clever-dumb"). The same linguistic control task (SC) was assigned to both generation tasks. For that purpose subjects had to subvocally change the order of two syllables of a logatome (e.g., "mora" should be changed to "ramo", neither of which are words in the German language). Such logatomes are defined as letter strings without semantic content but which obeyed the combination rules of phonemes and graphemes of the language in question, here German. All of logatomes consisted of four letters in the form consonant-vowel-consonantvowel. This control task was separately performed in both paradigms. Accordingly, $\mathrm{SC}_{1}$ refers to the first and $\mathrm{SC}_{2}$ to the second paradigm.

In dependence on the generation tasks two semantic congruency decision tasks were performed: a verb congruency decision task (VD) and an antonym decision task (AD). Here participants had to indicate by a button press if two words were either semantically related or not. In the third paradigm the verbdecision condition (VD) was conducted. Here participants indicated by button press if a presented noun matched a verb semantically. In the fourth paradigm the $\mathrm{AD}$ condition requires a button press depending on whether the two adjectives are antonyms or not. In the control task for the VD and AD condition two letter rows composed of four differently colored consonants (either blue or yellow) were presented. For each trial the participants had to discover a rule which associated the colors blue or yellow to each of the two consonants and to decide by button press if this rule is conformed to or violated. In the different trials the colors blue and yellow remain the same while the two used consonants changed in each trial. This control condition was performed separately in the third paradigm $\left(\mathrm{CON}_{1}\right)$ as well as in the fourth paradigm $\left(\mathrm{CON}_{2}\right)$.

All paradigms were conducted in block designs with a random alternation between experimental and control conditions and each block lasted $20 \mathrm{~s}$. A fixation phase, during which the participant viewed a central cross on the display, was inserted between the two conditions of every paradigm lasting $10 \mathrm{~s}$. In all language-generation conditions the participants were instructed to produce covert speech. The order of the four different paradigms was randomized. In the following analysis the four paradigms were processed as separate sessions. This method was used because it is not possible to conduct all conditions in an interleaved block-design fashion. Each paradigm required $10 \mathrm{~min}$ and 
$12 \mathrm{~s}$ to complete. The participant was instructed to rest within the MR-scanner between sessions.

\section{3. $f M R I$ acquisition}

Scanning was performed on a Siemens Allegra 3-Tesla scanner with a single channel head-coil system. Functional images were acquired using a T2* weighted gradient EPI $\left(T R=2000 \mathrm{~ms}, T E=30 \mathrm{~ms}, 34\right.$ slices, $F o V=192 \mathrm{~mm}$, flip angle $=90^{\circ}$, $3 \times 3 \times 3 \mathrm{~mm}^{3}$ voxel size, 306 scans per session). A high resolution T1 weighted image was also performed for each subject $(\mathrm{TR}=2300 \mathrm{~ms}$, TE $=2.91 \mathrm{~ms}, 160$ slices, FoV $=256 \mathrm{~mm}$, flip angle $=9^{\circ}, 1 \times 1 \times 1 \mathrm{~mm}^{3}$ voxel size) .

\subsection{MRI data analysis}

Imaging data was conducted by using the SPM5 software package (SPM5, Wellcome Department of Imaging Neuroscience, London, UK) running under Matlab7.1 (Mathworks, Sherborn, MA, USA). The preprocessing steps included motion correction (realignment), co-registration, segmentation and after that normalization to the MNI-Standard brain ICBM 152 which is based on 152 brains and was created by the Montreal Neurological Institute (MNI) with a $2 \times 2 \times 2$ resolution. In a final step the images were spatially smoothed by an isotropic Gaussian Kernel with FWHM of $6 \mathrm{~mm}$.

For the inference statistical analysis on the basis of the General Linear Model individual design matrices were generated. A matrix consisted of 4 sessions with two regressors while each session included an experimental (VG or AG or VD or $\mathrm{AD}$ ) and the corresponding control condition $\left(\mathrm{SC}_{1}, \mathrm{SC}_{2}, \mathrm{CON}_{1}, \mathrm{CON}_{2}\right)$. A boxcar model function of every condition was convoluted with the canonical hemodynamic response function (hrf). The fixation period was not explicitly modeled as a separate regressor but is included in the implicit baseline (BL). T-tests were computed for every voxel to compare the BOLD activity and the $\beta$-weights of the two different conditions in every paradigm.

The calculated contrasts of the second-level random effects analysis are [VG-BL], [VG-SC 1 , [VD-BL] and [VD-CON 1 . The level of significance on voxel level was $P_{\text {uncor }}=0.001$. A $P_{\text {cor }}$-value of 0.05 was used on the cluster level. All brain areas associated with primary language functions were anatomically defined as region of interest (ROI) by using the Anatomical Automatic Labeling (AAL)-atlas (Tzourio-Mazoyer et al., 2002). These areas included the bilateral pars opercularis, pars triangularis, the frontal inferior gyrus (IFG), superior temporal gyrus, angular and supramarginal gyrus. A ROI analysis was conducted by applying the MarsBar toolbox implemented in SPM5. Percent signal change for the contrasts [VG-BL], $[A G-B L],[V D-B L]$ and $[A D-B L]$ within the left pars opercularis and pars triangularis were calculated by averaging the time course of each voxel to handle the problem of multiple comparison (Brett, Anton, Valabregue, \& Poline 2002). Twotailed Wilcoxon matched pairs signed ranks tests were executed to test the differences of percent signal change between the contrasts [VG-BL] and [VD-BL] and between the contrasts $[\mathrm{AG}-\mathrm{BL}]$ and $[\mathrm{AD}-\mathrm{BL}]$ for significance $(P<0.05)$.

\subsection{Assessment of lateralization indices}

The lateralization indices (LI) describe the measurement of the portion of active voxels in the left and right hemisphere. The values range between 1 and -1 , whereas 1 stands for complete left and -1 for complete right hemispheric language dominance. For a discussion of the problems concerning the use of the LI see Seghier (2008).

$\mathrm{LI}=\left(N_{l}-N_{r}\right) /\left(N_{l}+N_{r}\right) ; N_{l}$ is the number of voxels active in the left hemisphere; $N_{r}$ is the number of voxels active in the right hemisphere.

ROIs described earlier were considered for LI analysis. A small volume correction was carried out because the voxel statistic only refers to the ROIs. All active voxels in the ROIs for $P_{\text {uncor }}<0.001$ went into the calculation of the LI. The LI-based contrasts and conjunctions listed in Table 1 were calculated for each participant separately. A conjunction analysis of two contrasts has the null hypothesis that each voxel is not activated significantly in the one or the other contrast (for further information see Friston, Penny, \& Glader, 2005).

The aim is to reveal progressively the effects of (i) conjunction analysis, (ii) linguistic control tasks and (iii) their combination on the LIs. Nonparametric

Table 1

Different contrasts and conjunctions used to calculate the LIs for every subject.

\begin{tabular}{ll}
\hline Paradigms 1 and 2 & Paradigms 3 and 4 \\
\hline$[V G-B L]$ & \\
{$[A G-B L]$} & \\
{$\left[V G-S C_{1}\right]$} & \\
{$[V G-B L] \cap[A G-B L]$} & \\
{$\left[V G-S C_{1}\right] \cap\left[A G-S C_{2}\right]$} & {$\left[V D-C O N_{1}\right] \cap\left[A D-C O N_{2}\right]$}
\end{tabular}

Wilcoxon matched pairs signed ranks tests (two-tailed) were used to test whether two medians of the absolute LIs differ significantly. The significance level was set at $P<0.05$.

\section{Results}

\subsection{Behavioral results}

The average reaction time for the verb decision (VD) condition was $1177.8 \mathrm{~ms}(\mathrm{SD}=150.42 \mathrm{~ms})$. Compared with the average reaction time (RT) of the control condition $\mathrm{CON}_{1} 1498.64 \mathrm{~ms}$ $(\mathrm{SD}=128.25)$ the Wilcoxon-test showed significantly lower RTs for VD than for $\mathrm{CON}_{1}$ (Wilcoxon- $Z=-2.89 ; P=0.003$ ). The same effect could be observed for a comparison between the results of the $\mathrm{AD}$ and $\mathrm{CON}_{2}$ conditions (Wilcoxon $Z=-2.8 ; P=0.005$ ). Accordingly, the average RT of the AD condition was $1285.1 \mathrm{~ms}$ $\left(\mathrm{SD}=200.3 \mathrm{~ms}\right.$ ) and the average RT of the $\mathrm{CON}_{2}$ condition was $1453.9 \mathrm{~ms}(\mathrm{SD}=108.0 \mathrm{~ms})$. There was no significant difference in the accuracy of the tasks. The mean percentage of correct responses was $95.0 \%(\mathrm{SD}=2.8 \%)$ for $\mathrm{VD}$ and $94.6 \%(\mathrm{SD}=3.2 \%)$ for $\mathrm{CON}_{1}$. For $\mathrm{AD}$ the fraction of correct responses was $94.3 \%$ $(\mathrm{SD}=2.9)$, for the corresponding control condition $\mathrm{CON}_{2} 95.8 \%$ $(\mathrm{SD}=2.4 \%)$.

\subsection{Activity in Broca's area for semantic generation and semantic decision tasks}

As shown in Fig. 1 the mean percent signal change of the ROI analysis of the left pars opercularis and triangularis for the contrast between the verb generation task and baseline $(M=0.476 ; \mathrm{SD}=0.140)$ was higher than for the percent signal change analysis for the contrast between verb decision and baseline $(M=0.318 ; \mathrm{SD}=0.147)$. This effect was significant (Wilcoxon $Z=-2.93 ; P=0.003$ ). The contrast between antonym generation and baseline $(M=0.449 ; \mathrm{SD}=0.148)$ revealed a higher mean percent signal change than did the contrast between antonym decision and baseline $(M=0.292 ; \mathrm{SD}=0.187)$ in the left pars opercularis and triangularis. The Wilcoxon test was also significant (Wilcoxon $Z=-2.58 ; P=0.01$ ).

\subsection{Results of the analysis of the lateralization indices}

The individual LIs for every subject are shown in Figs. 2 and 3. The means of the absolute LIs assessed by different contrasts and analysis methods are represent in Fig. 4. Furthermore we illustrate in Fig. 5 the absolute LIs as a function of the statistical threshold.

The means of the absolute LIs were $0.445(S D=0.262)$ for the contrast verb generation compared to baseline [VG-BL] and 0.453 $(S D=0.266)$ for the contrast antonym generation compared to baseline conditions ([AG-BL]). There was a significant difference of the central tendency between the LIs based on these single contrasts and the LIs based on the conjunction of these contrasts [VG-BL] $\cap$ AG-BL] with $M=0.507(S D=0.278)$. For the comparison between $[\mathrm{VG}-\mathrm{BL}]$ and $[\mathrm{VG}-\mathrm{BL}] \cap[\mathrm{AG}-\mathrm{BL}]$ Wilcoxon $Z$ was -3.06 with a corresponding $P$ value of 0.002 and for the comparison between $[\mathrm{AG}-\mathrm{BL}]$ and $[\mathrm{VG}-\mathrm{BL}] \cap[\mathrm{AG}-\mathrm{BL}]$ Wilcoxon $Z$ was -2.90 with $P=0.004$.

For the absolute LIs calculated on the basis of the conjunction between verb generation and antonym generation with respect to baseline [VG-BL] $\cap \mathrm{AG}-\mathrm{BL}](M=0.507 ; \mathrm{SD}=0.278)$ and the contrast between the verb generation and the linguistic syllable exchange control tasks [VG-SC $C_{1}$ ( $\left.M=0.678 ; \mathrm{SD}=0.295\right)$ the LIs of the contrast [VG-SC1] yielded significantly higher LIs than the LIs of the conjunction $[\mathrm{VG}-\mathrm{BL}] \cap[\mathrm{AG}-\mathrm{BL}]$ (Wilcoxon $Z=-2.68$; 

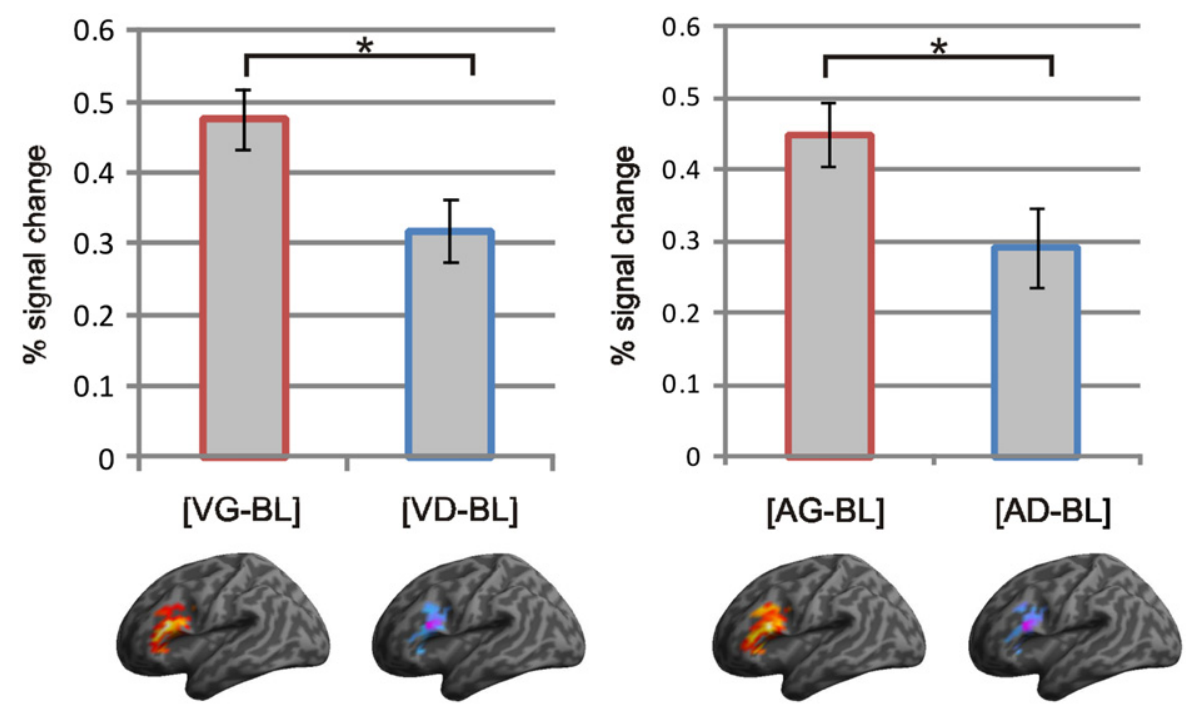

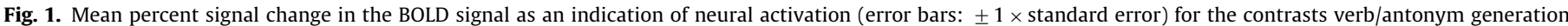

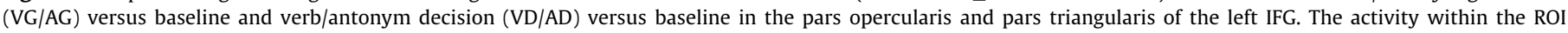
(after small volume correction) is shown on the left hemisphere of the MNI standard brain.

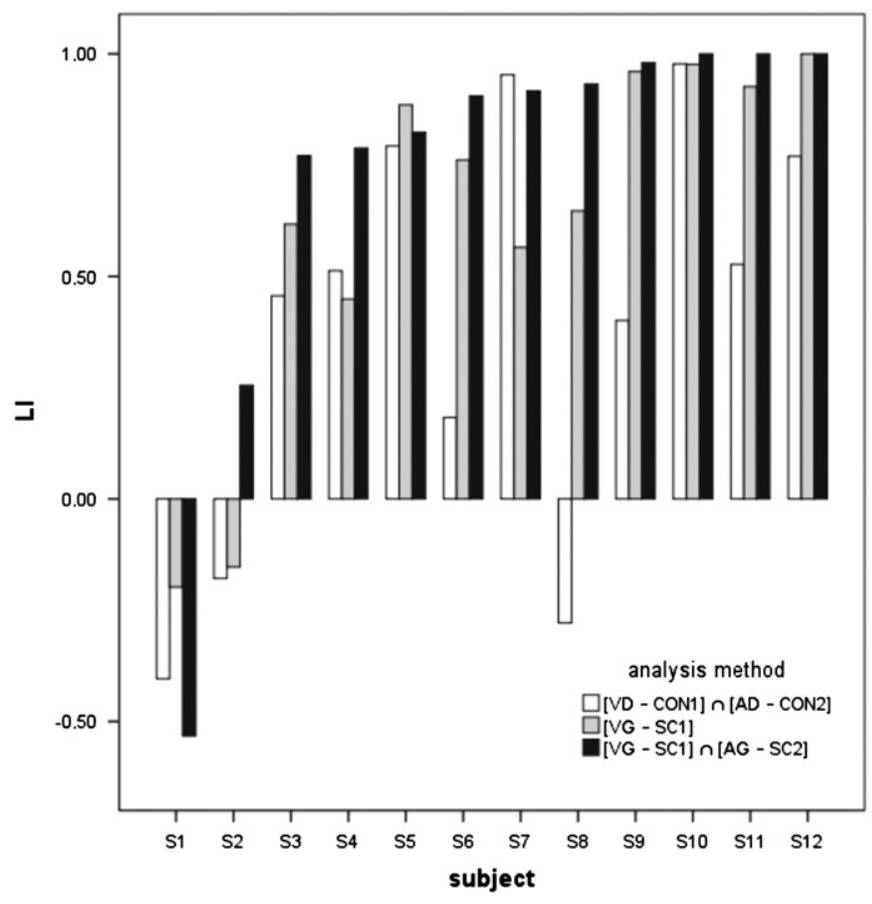

Fig. 2. Lateralisation indices (LIs) of the single contrast of verb generation (VG) versus syllable exchange (SC1), the conjunction of verb and antonym generation (AG) versus syllable exchange (SC1/SC2) and the conjunction of verb and antonym decision (VD/AD) versus consonant-color-task (CON1/CON2) for every subject.

$P=0.008)$. The comparison of the absolute LIs showed that conjunction consisting of the two differential contrasts for verb and antonym generation tasks $\left[\mathrm{VG}-\mathrm{SC}_{1}\right]$ and $\left[\mathrm{AG}-\mathrm{SC}_{2}\right](M=0.826$; $\mathrm{SD}=0.225$ ) were significantly larger (Wilcoxon $Z=2.67 ; P=0.008$ ) than the LIs of the single contrast [VG-SC $C_{1}(M=0.678$; $\mathrm{SD}=0.295$ ).

We compared the absolute LIs for the conjunction of the generation tasks to the conjunction of the decision tasks. The highest absolute LIs were observed for the conjunction analysis of the generation tasks, when both generation paradigms were compared to control condition ([VG-SC $\left.{ }_{1}\right] \cap\left[\mathrm{AG}-\mathrm{SC}_{2}\right] ; M=0.826$; $\mathrm{SD}=0.225$ ). The conjunction analysis of the decision paradigms

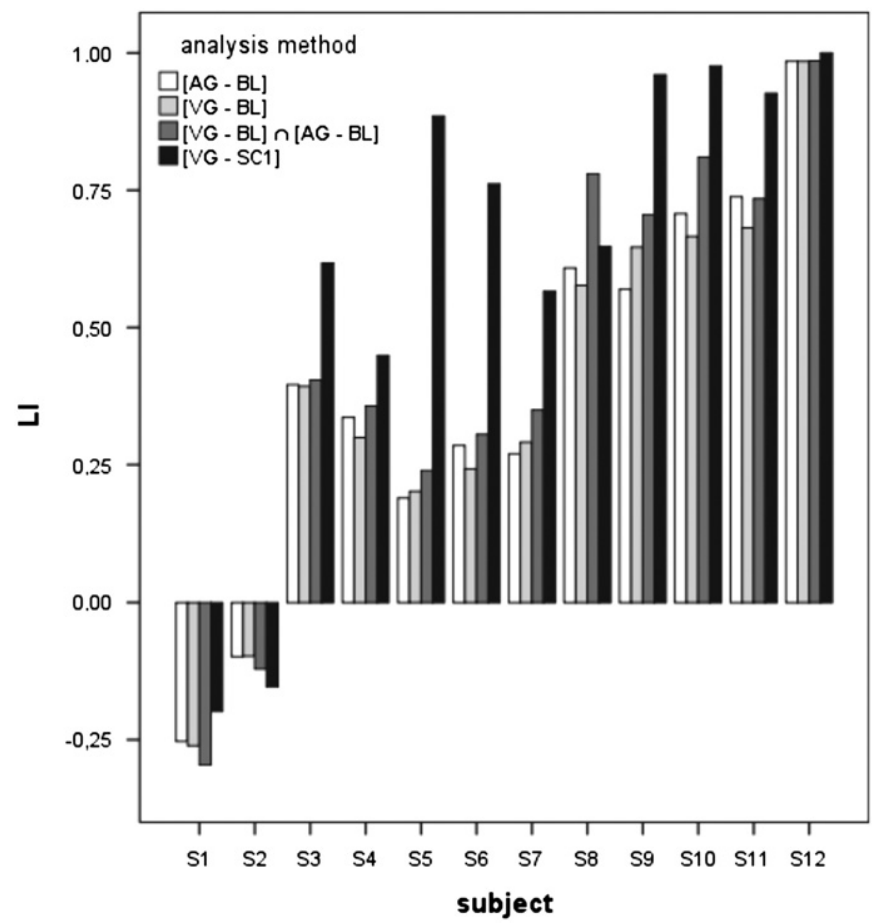

Fig. 3. Lateralization indices (LIs) of the baseline (BL) contrasts with verb and antonym generation (VG/AG), the conjunction of these two contrasts and the contrast verb generation (VG) versus syllable changing (SC1) for every subject.

(including both decision paradigms compared to control tasks [VD- $\left.\mathrm{CON}_{1}\right] \cap\left[\mathrm{AD}-\mathrm{CON}_{2}\right]$ ) led to significant lower absolute LIs; $M=0.536 ; \mathrm{SD}=0.278$ (Wilcoxon $Z=-2.82$ und $P=0.005$ ).

\section{Discussion}

\subsection{Broca's area activation induced by semantic decision and} generation tasks

In the present study, we focused on the differences between linguistic generation and decision tasks. The comparison of the 

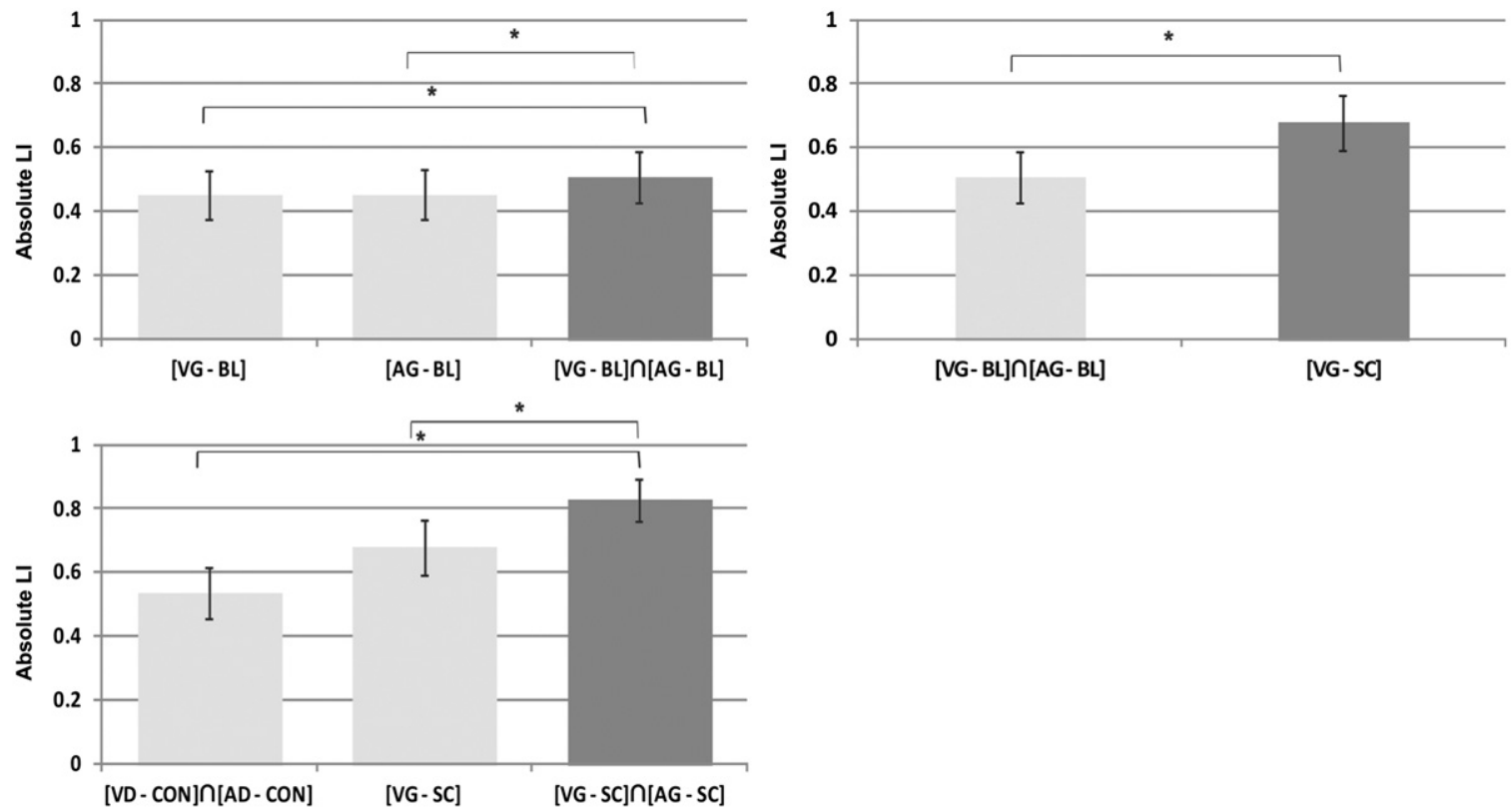

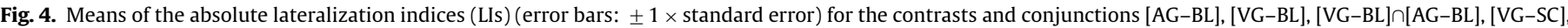

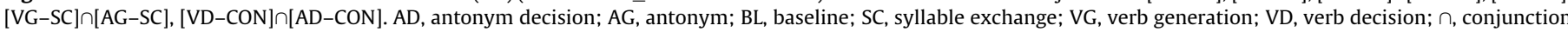
of two contrasts. Note: the asterisks refer to the significance of the nonparametric Wilcoxon test.

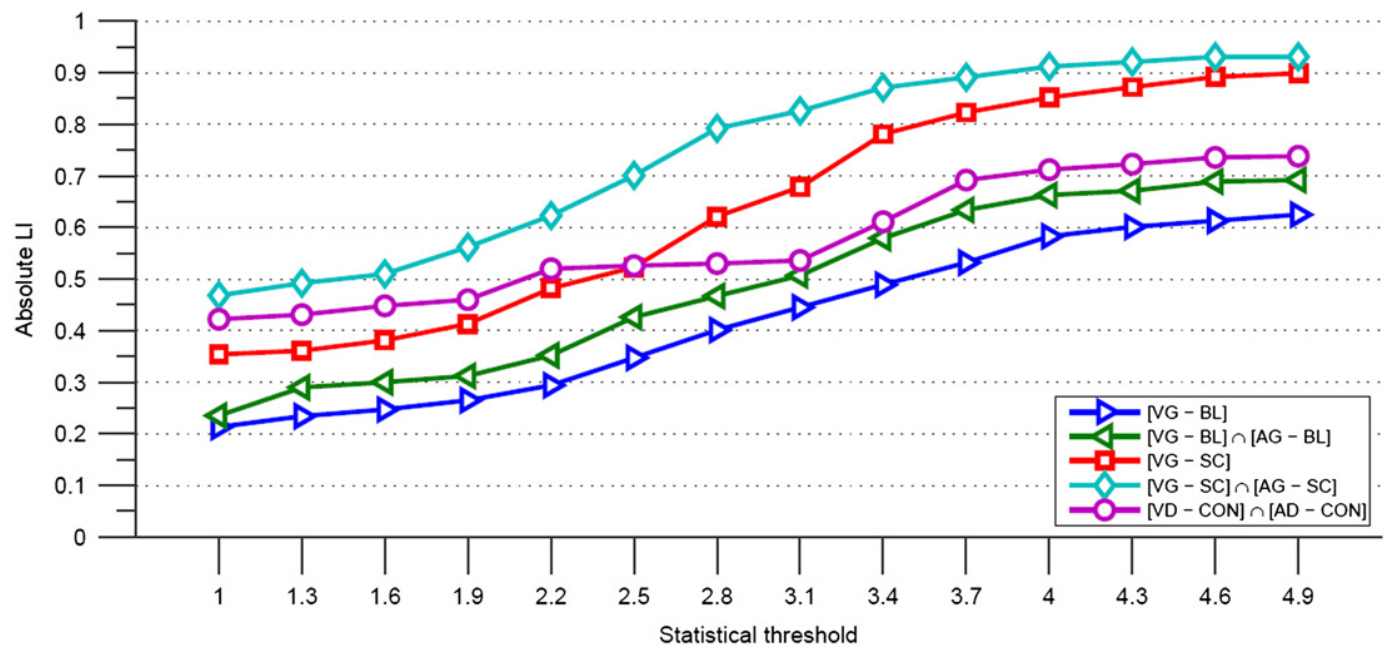

Fig. 5. Means of the absolute lateralization indices (LIs) as function of the statistical threshold ( $T$ score)

magnitude of activation between the semantic word generation tasks (verb generation, VG, and antonym generation, AG) and their corresponding decision tasks (verb decision, VD, and antonym decision, AD) revealed a significantly higher percent signal change for the generation task in the pars opercularis and pars triangularis of the left IFG (BA 44 and BA 45; see Fig. 1). Thompson-Schill, D’Esposit, Aguirre, and Farah (1997) and Thompson-Schill et al. (1998) emphasize the role of left IFG for the selection of semantic knowledge. The authors assume that the IFG does not regulate semantic retrieval per se but participates in the selection of semantic features out of a set of rivaling alternatives. Badre and Wagner (2007), Martin and Cheng (2006) and Wagner, Paré-Blagoev, Clark, and Poldrack (2001) challenge the selectivity dependent activity of the IFG and propagate an increase of activity as a result of semantic retrieval demands and the level of control required during retrieval when selection demands stay constant.

In the generation tasks of our study, subjects did not need to pay attention to selective features like color, shape or size. The only predetermined selectivity applies to the morpho-syntactic specifications regarding the word form (i.e., whether the generated word was a verb for VG or an adjective for AG tasks) and the global semantic congruence. In the case of the decision tasks we assume that a global comparison of semantic congruence took place. Because the same nouns and adjectives were used in the generation and decision tasks, the concept of selectivity proposed by Thompson-Schill et al. (1997) is not relevant for our results. Rather we would like to focus on the demands of semantic retrieval specified by the different tasks. These tasks place different demands on top-down control mechanisms. The generation tasks do not require subjects to retrieve one or more words, which are most closely semantically related to a previously presented word, but rather they are asked to determine an appropriate verb for the particular item without any limitation. In contrast to decision tasks (VD and $A D$ ), which require the determination and evaluation of the distance of two words within the semantic space, during the generation tasks additional processes of selection and generation of a word take place. It follows 
that generation tasks are more challenging than decision tasks. Overall, semantic word generation tasks demand specific aspects of an explicit semantic working memory task including the retrieval of information from semantic memory and its manipulation to generate an appropriate verb. Explicit working memory tasks consistently activate prefrontal brain areas (Bunge, Ochsner, Desmond, Glover, \& Gabrieli, 2001; Hautzel et al., 2002; Rypma, Prabhakaran, Desmond, Glover, \& Gabrieli, 1999). In summary the prominent activation of the pars opercularis (BA 44) and pars triangularis (BA 45) in our study underlines the usefulness of semantic generation tasks for localizing this region for example in preoperative surgical mapping with fMRI.

\subsection{Effects of different language paradigms and analysis methods on the lateralization index}

\subsubsection{Semantic decision or semantic generation?}

Because generation tasks do not allow for the control of behavioral performance decision tasks were included in our experimental design. We asked whether semantic decision tasks, which include the recording of behavioral data, led to LIs that are comparable to those based on semantic word generation tasks. To this end we compared the magnitude of LIs of conjunction between both generation contrasts $\left[\mathrm{VG}-\mathrm{SC}_{1}\right] \cap\left[\mathrm{AG}-\mathrm{SC}_{2}\right]$ with the magnitude of LIs of the decision contrasts [VD-CON $\left.{ }_{1}\right] \cap$ $\left[\mathrm{AD}-\mathrm{CON}_{2}\right]$. The generation conjunction yielded to significant greater absolute LIs than the LIs based on the conjunction of the decision contrasts. Nevertheless the LIs of the decision contrasts seem to be more reliable in terms of threshold robustness than LIs based on generation contrasts (see Fig. 5). In this context Binder (2011) pointed out that semantic decision tasks can improve significantly the prediction of postoperative memory and language performance in clinical settings.

The result of lower LIs for the decision tasks conjunction compared to the generation task conjunction is not unexpected because studies which use semantic or syntactic decision tasks often found activation of the right hemispheric counterparts of left hemispheric structures, which are primarily associated with language processing. Using PET, Moro et al. (2001) investigated the neuronal correlates of syntactic, morpho-syntactic, and phonotactic processes. For this purpose they created decision tasks, in which the subjects were asked to detect sentences with errors in one of these three linguistic domains. For each of these three conditions they found not only left-sided frontal and temporal activation but also activation of the right IFG, STG and the medial temporal gyrus. Also the studies of Dapretto and Bookheimer (1999) and Friederici, Rüschemeyer, Hahne, and Fiebach (2003), which include semantic judgments, revealed activation of right temporoparietal and frontal brain areas. Taken together with the present results it seems that the counterparts of the classical language areas (Broca and Wernicke) in the non-dominant hemisphere support conscious abstraction on the level of metalinguistic knowledge (Moro et al., 2001). This meta-linguistic knowledge is required for acceptability ratings regarding semantic or syntactic dimensions. Along these lines, our semantic decision tasks require enhanced demands on semantic metalinguistic knowledge.

\subsubsection{Conjunction of activations evoked by two semantic generation} tasks

The conjunction of the contrasts for the generation tasks

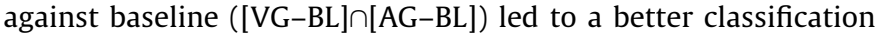
of LIs than those based on the single contrasts ([VG-BL] or [AG-BL]) alone. The study by Ramsey et al. (2001) revealed similar results. Those authors based their conclusions on the conjunction of several contrasts from language tasks versus a passive rest condition with open eyes. They argue that a single contrast from a language task versus passive rest includes many processes, which are not critical for language. Furthermore they assume that the conjunction of two or more language tasks does not detect the task specific neuronal correlates but rather the activity of neuronal structures, which form the basis of all language tasks. These structures should be consistent with areas revealed by IES and may be more lateralized.

\subsubsection{Control condition or conjunction analysis?}

Appropriate control conditions are essential for studies using fMRI or PET techniques. A good control condition should contain all processes required by the experimental condition except for the process of interest. An argument often put forth in this context is that in the case of clinical assessment of language dominance the focus does not lie on specific subprocesses of language but rather on general language functions in the left or right hemisphere. There are some latter studies that deal with the problem of adequate control conditions (Binder, 2011) or with the use of semantic generation and decision paradigms (Jansen et al., 2006). Our aim was not only to investigate the effects of cognitive high-level control conditions but we also focus on the nonautomatic linguistic domain as a control condition. The activation maps in Fig. 6 illustrate how the control conditions lead to a decrease of activation in non-eloquent brain regions. Generally the effect of the control condition is smaller for the generation than for the decision tasks. In the case of the verb generation tasks the control condition eliminates activation of non-eloquent brain

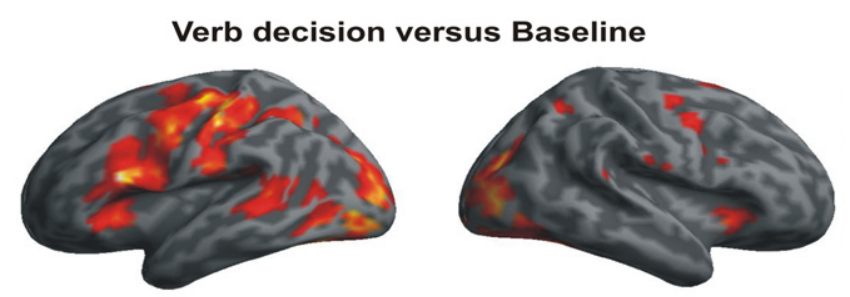

Verb decision (VD) versus Consonant-Color-Task
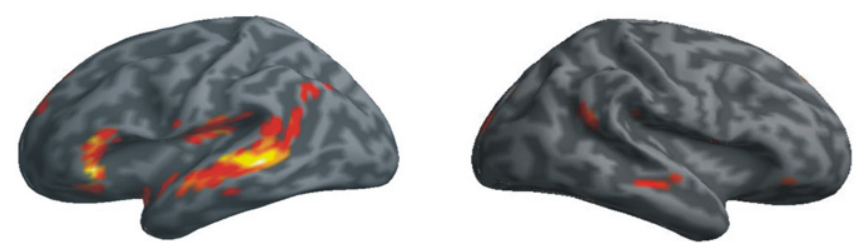

Verb generation (VG) versus Baseline (BL)
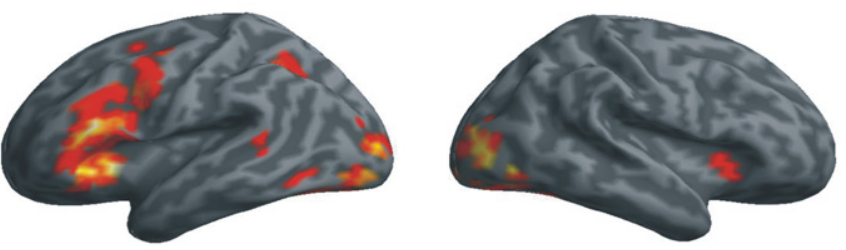

Verb generation (VG) versus Syllable Exchange (SC)
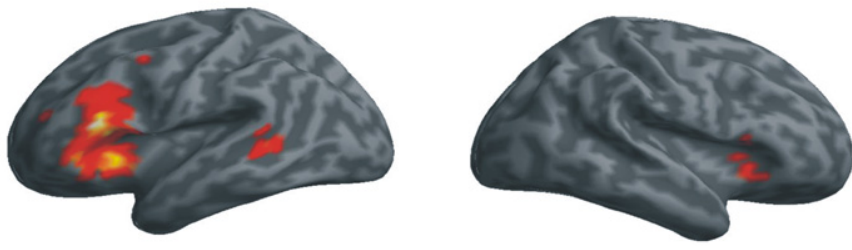

Fig. 6. Activation maps of the contrasts verb generation (VG) versus baseline (BL), verb generation versus syllable exchange (SC) verb decision versus baseline and verb decision versus consonant-color-task (CON) with $P_{\text {uncor }}=0.001$ on voxel level. 
areas that are irrelevant for our analysis. Prefrontal and temporal activation remains nearly unaffected. This reduction enables us to use more global ROIs as suggested by Seghier (2008) and avoids the problem of inter-individual differences in the anatomical location and extension of neural functional units. However, in addition to the deactivation of non-eloquent brain regions the verb decision control task leads to a pronounced increase of left frontal and at the same time to a moderate decrease of superior temporal regions. The control conditions of the decision tasks appear to hinder the discovery of general hemispheric language dominance, although care needs to be taken when inferring the extent of the individual LIs and their mean value from the group level t-map.

The contrast of a verb generation task (VG) versus a phonological syllable exchange task (SC) led to significantly greater absolute LIs compared to the values calculated by the conjunction between verb and antonym generation tasks ([VG-BL] $[\mathrm{AG}-\mathrm{BL}]$ ). We interpret these findings in terms of eliminating the effects of non-essential cognitive and sensory processes (attention, memory, and presentation modality). These nonspecific language processes could be responsible for more widespread brain-imaging activations compared to eloquent areas revealed by IES (Bookheimer et al., 1997; FitzGerald et al., 1997). It follows that the conjunction analysis proposed by Ramsey et al. (2001) and Rutten et al. (2002a, 2002b) cannot be an adequate procedure since conjunction analysis only reveals the areas of overlap between the analyzed contrasts. The adequacy of the semantic word generation tasks is based on the pronounced effort required for the controlled semantic retrieval (Badre \& Wagner, 2002) and the neuronal correlates of this process in the IFG of the language dominant hemisphere. In our case we used a control condition consisting of pseudo-words evoking phonological but not semantic processing (Siok, Jin, Fletcher, \& Tan, 2003), thereby isolating predominantly processes of semantic retrieval and semantic analysis, to reflect general hemispheric-dominance in language processing. The greater absolute LIs of the contrast [VG-SC $\mathrm{C}_{1}$ ] compared to the conjunction between verb generation and

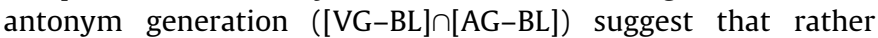
highly specific processes of the language tasks than language nonspecific processes led to pronounced bilateral activation patterns with the result of poorly classifying LIs.

\subsubsection{Control condition and conjunction analysis}

We calculated LIs on the basis of the conjunction of the two contrasts of the word generation tasks in each case versus a linguistic control task ( $\left.\left[\mathrm{VG}-\mathrm{SC}_{1}\right] \cap\left[\mathrm{AG}-\mathrm{SC}_{2}\right]\right)$ and compared these LIs with the LIs based on a single contrast ([VG-SC $\left.C_{1}\right]$ ). The conjunction with an additional generation contrast improved the goodness of classification in terms of unambiguousness of lateralized language function. This result and the findings discussed above indicate that it is necessary to choose an appropriate control condition, which eliminates general processes within the linguistic network. In addition the conjunction of two different semantic word generation tasks with linguistic control condition partials out the effects of highly task-specific processes. This combination of both procedures leads to more unambiguous LIs.

Despite the high absolute LI values (for the combined conjunction control task analysis of the semantic word generation tasks 10 of 12 subjects had LIs greater than 0.7 ) the validity of the laterality remains to be determined. Since the intrinsic hemispheric language dominance of the participants is unknown, even for the three participants with a LI of +1 , we cannot definitely determine whether they are truly left language lateralized. For this purpose methods that induce a temporary loss of function are necessary. These procedures are the Wada test or IES during brain surgery. Both methods are highly invasive and require medical indication. For that reason this question has to be settled in a patient population by a comparison of language dominance revealed by these valid, clinical methods and the dominance assessed by fMRI.

The external validity is an issue that arises when the findings of studies with healthy participants should be generalized to a clinical population. An important question in this context is whether patients are able to perform the proposed cognitive high-level control tasks. Our experience indicates that most neurosurgical patients can perform these tasks. When they have difficulties they often fail in all tasks. In these cases we have to reduce the cognitive effort and use tasks involving more automated linguistic processes like word reading or picture naming.

In summary these findings support the notion that in a clinical setting the use of conjunction analysis and control tasks can help to achieve LIs that reflect clearly the general language dominance. Although the decision tasks lead to more bilateral activation especially of temporal language associated regions than the generation tasks, the resulting LIs seem to be more robust over a wide range of $T$-values and bring to bear the benefit of behavioral control. As expected in comparison to the decision tasks the generation tasks lead to significantly more activation of BA 44 and 45 . So in a clinical setting it should be advantageous to apply more than one language paradigm with linguistic control conditions and to take account of the intersection of their activation when determining hemispheric language dominance by fMRI.

\section{References}

Adcock, J. E., Wise, R. G., Oxbury, J. M., Oxbury, S. M., \& Mattews, P. M. (2003) Quantitative fMRI assessment of the differences in lateralization of languagerelated brain activation in patients with temporal lobe epilepsy. NeuroImage 18(2), 423-438.

Amunts, K., Weiss, P. H., Mohlberg, H., Pieperhoff, P., Eickhoff, S., \& Gurd, J. M. (2004). Analysis of neural mechanisms underlying verbal fluency in cytoarchitectonically defined stereotaxic space-the roles of Brodmann areas 44 and 45 Neurolmage, 22(1), 42-56.

Badre, D., \& Wagner, A. D. (2002). Semantic retrieval, mnemonic control, and prefrontal cortex. Behavioral and Cognitive Neuroscience Reviews, 1(3), 206-218.

Badre, D., \& Wagner, A. D. (2007). Left ventrolateral prefrontal cortex and the cognitive control of memory. Neuropsychologia, 45(13), 2883-2901.

Baillieux, H., Vandervliet, E. J., Manto, M., Parizel, P. M., De Deyn, P. P., \& Mariën, P. (2009). Developmental dyslexia and widespread activation across the cerebellar hemispheres. Brain and Language, 108(2), 122-132.

Binder, J. R., Frost, J. A., Hammeke, T. A., Bellgowan, P. S., Rao, S. M., \& Cox, R. W. (1999). Conceptual processing during the conscious resting state. A functional MRI study. Journal of Cognitive Neuroscience, 11(1), 80-95.

Binder, J. R. (2011). FMRI is a valid noninvasive alternative to Wada testing Epilepsy Behavior, 20(2), 214-222.

Bleich-Cohen, M., Hendler, T., Kotler, M., \& Strous, R. D. (2009). Reduced language lateralization in first-episode schizophrenia: An fMRI index of functional asymmetry. Psychiatry Research, NeuroImaging, 171(2), 82-93.

Bookheimer, S. Y., Zeffiro, T. A., Blaxton, T. A., Malow, B., Gaillard, W. D., Sato, S. et al. (1997). A direct comparison of PET activation and electrocortical stimulation mapping for language localization. Neurology, 48(4), 1056-1065.

Brannen, J. H., Badie, B., Moritz, C. H., Quigley, M., Meyerand, M. E., \& Haughton, V. M. (2001). Reliability of functional MR imaging with word-generation tasks for mapping Broca's area. American Journal of Neuroradiology, 22(9), 1711-1718.

Brett, M., Anton, J. L., Valabregue, R., \& Poline, J. -B. (2002). Region of interest analysis using an SPM toolbox. Presented at the 8th international conference on functional mapping of the human brain. Sendai, Japan. Vol. 16, No. 2 [abstract 497 available on CD-ROM in NeuroImage]

Bunge, S. A., Ochsner, K. N., Desmond, J. E., Glover, G. H., \& Gabrieli, J. D. (2001) Prefrontal regions involved in keeping information in and out of mind. Brain 124(10), 2074-2086.

Dapretto, M., \& Bookheimer, S. Y. (1999). Form and content: dissociating syntax and semantics in sentence comprehension. Neuron, 24(2), 427-432.

Engström, M., Ragnehed, M., Lundberg, P., \& Söderfeldt, B. (2004). Paradigm design of sensory-motor and language tests in clinical fMRI. Neurophysiologie Clinique/ Clinical Neurophysiology, 34(6), 267-277.

Fiez, J. A., Raichle, M. E., Balota, D. A., Tallal, P., \& Petersen, S. E. (1996). PET activation of posterior temporal regions during auditory word presentation and verb generation. Cerebral Cortex, 6(1), 1-10. 
FitzGerald, D. B., Cosgrove, G. R., Ronner, S., Jiang, H., Buchbinder, B. R., Belliveau, J. W., et al. (1997). Location of language in the cortex: A comparison between functional MR imaging and electrocortical stimulation. American Journal of Neuroradiology, 18(8), 1529-1539.

Friederici, A. D., Rüschemeyer, S. A., Hahne, A., \& Fiebach, C. J. (2003). The role of left inferior frontal and superior temporal cortex in sentence comprehension: Localizing syntactic and semantic processes. Cerebral Cortex, 13(2), 117-177.

Friston, K. J., Penny, W. D., \& Glader, D. E. (2005). Conjunction revisted NeuroImage, 25(3), 661-667.

Gaillard, W. D., Hertz-Pannier, L., Mott, S. H., Barnett, A. S., Lebihan, D., \& Theodore W. H. (2000). Functional anatomy of cognitive development: fMRI of verbal fluency in children and adults. Neurology, 54(1), 180-185.

Hautzel, H., Mottaghy, F. M., Schmidt, D., Zemb, M., Shah, N. J., Müller-Gärtner, H. W., et al. (2002). Topographic segregation and convergence of verbal, object, shape and spatial working memory in humans. Neuroscience Letters, 323(2) $156-160$.

Hertz-Pannier, L., Chiron, C., Jambaqué, I., Renaux-Kieffer, V., Van de Moortele, P. F., Delalande, O., et al. (2002). Late plasticity for language in a child's nondominant hemisphere: A pre- and post-surgery fMRI study. Brain, 125(2), 361-372.

Hunter, K. E., Blaxton, T. A., Bookheimer, S. Y., Figlozzi, C., GaillardW., D., Grandin, C., et al. (1999). O-15 water positron emission tomography in language localization: A study comparing positron emission tomography visual and computerized region of interest analysis with the Wada test. Annals of Neurology, 45(5), 662-665.

Jansen, A., Menke, R., Sommer, J., Förster, A. F., Bruchmann, S., Hempleman, J., et al. (2006). The assessment of hemispheric lateralization in functional MRIrobustness and reproducibility. NeuroImage, 33(1), 204-217.

Kho, K. H., Leijten, F. S., Rutten, G. J., Vermeulen, J., Van Rijen, P., \& Ramsey, N. F. (2005). Discrepant findings for Wada test and functional magnetic resonance imaging with regard to language function: Use of electrocortical stimulation mapping to confirm results. Case report. Journal of Neurosurgery, 102(1), 169-173.

Kleinhans, N. M., Müller, R., Cohenc, D. N., \& Courchesnee, E. (2008). Atypical functional lateralization of language in autism spectrum disorders. Brain Research, 1221, 115-125.

Knecht, S., Dräger, B., Deppe, M., Bobe, L., Lohmann, H., Flöel, A., et al. (2000) Handedness and hemispheric language dominance in healthy humans. Brain. 123(12), 2512-2518.

Knecht, S., Jansen, A., Frank, A., van Randenborgh, J., Sommer, J., Kanowski, M., et al. (2003). How atypical is atypical language dominance?. Neurolmage, 18(4), 917-927.

Li, X., Branch, C. A., Ardekani, B. A., Bertisch, H., Hicks, C., \& DeLisi, L. E. (2007). fMR study of language activation in schizophrenia, schizoaffective disorder and in individuals genetically at high risk. Schizophrenia Research, 96(1-3), 14-24.

Lindell, A. K. (2006). In your right mind: Right hemisphere contributions to language processing and production. Neuropsychology Review, 16, 131-148.

Loring, D. W., Meador, K. J., Lee, G. P., Murro, A. M., Smith, J. R., Flanigin, H. F., et al. (1990). Cerebral language lateralization: Evidence from intracarotid amobarbital testing. Neuropsychologia, 28(8), 831-838.

Martin, R. C., \& Cheng, Y. (2006). Selection demands versus association strength in the verb generation task. Psychonomic Bulletin and Review, 13(3), 396-401.

Milne, R. D., Syngeniotis, A., Jackson, G., \& Corballis, M. C. (2002). Mixed lateralization of phonological assembly in developmental dyslexia. Neurocase 8(3), 205-209.

Moro, A., Tettamanti, M., Perani, D., Donati, C., Cappa, S. F., \& Fazio, F. (2001). Syntax and the brain: Disentangling grammar by selective anomalies. NeuroImage, 13(1), 110-118.

Newman, S. D., \& Twieg, D. (2001). Differences in auditory processing of words and pseudowords: An fMRI study. Human Brain Mapping, 14(1), 39-47.

Ojemann, G., Ojemann, J., Lettich, E., \& Berger, M. (1989). Cortical language localization in left, dominant hemisphere. An electrical stimulation mapping investigation in 117 patients. Journal of Neurosurgery, 108(2), 411-421.

Oldfield, R. C. (1971). The assessment and analysis of handedness: The Edinburgh inventory. Neuropsychologia, 9(1), 97-114.

Pujol, J., Deus, J., Losilla, J. M., \& Capdevila, A. (1999). Cerebral lateralization of language in normal left-handed people studied by functional MRI. Neurology, 52(5), 1038-1043.
Ramsey, N. F., Sommer, I. E., Rutten, G. J., \& Kahn, R. S. (2001). Combined analysis of language tasks in fMRI improves assessment of hemispheric dominance for language functions in individual subjects. NeuroImage, 13(4), 719-733.

Rasmussen, T., \& Milner, B. (1977). The role of early left-brain injury in determining lateralization of cerebral speech functions. Annals of the New York Academy of Sciences, 299, 355-369.

Riecker, A., Ackermann, H., Wildgruber, D., Meyer, J., Dogil, G., Haider, H., et al. (2000). Articulatory/phonetic sequencing at the level of the anterior perisylvian cortex: A functional magnetic resonance imaging (fMRI) study. Brain and Language, 75(2), 259-276.

Risse, G. L., Gates, J. R., \& Fangmana, M. C. (1997). A reconsideration of bilateral language representation based on the intracarotid amobarbital procedure. Brain and Cognition, 33(1), 118-132.

Rutten, G. J., Ramsey, N. F., van Rijen, P. C., Alpherts, W. C., \& van Veelen, C. W. (2002a). FMRI-determined language lateralization in patients with unilateral or mixed language dominance according to the Wada test. NeuroImage, 17(1), 447-460.

Rutten, G. J., Ramsey, N. F., van Rijen, P. C., Noordmans, H. J., \& van Veelen, C. W. (2002b). Development of a functional MRI protocol for intraoperative localization of critical temporoparietal language areas. Annals of Neurology, 51(3), 350-360.

Rypma, B., Prabhakaran, V., Desmond, J. E., Glover, G. H., \& Gabrieli, J. D. (1999). Load-dependent roles of frontal brain regions in the maintenance of working memory. NeuroImage, 9(2), 216-226.

Schäffler, L., Lüders, H. O., \& Beck, G. J. (1996). Quantitative comparison of language deficits produced by extraoperative electrical stimulation of Broca's, Wernicke's, and basal temporal language areas. Epilepsia, 37(5), 463-475.

Seghier, M. L. (2008). Laterality index in functional MRI: Methodological issues. Journal of Magnetic Resonance Imaging, 26(5), 594-601.

Siok, W. T., Jin, Z., Fletcher, P., \& Tan, L. H. (2003). Distinct brain regions associated with syllable and phoneme. Human Brain Mapping, 18(3), 201-207.

Sommer, I., Ramsey, N., Kahn, R., Aleman, A., \& Bouma, A. (2001). Handedness, language lateralisation and anatomical asymmetry in schizophrenia: Metaanalysis. The British Journal of Psychiatry, 178, 344-351.

Szaflarski, J. P., Holland, S. K., Schmithorst, V. J., \& Byars, A. W. (2006). fMRI study of language lateralization in children and adults. Human Brain Mapping, 27(3), 122-202.

Thompson-Schill, S. L., D’Esposit, M., Aguirre, K., \& Farah, M. (1997). Role of left inferior prefrontal cortex in retrieval of semantic knowledge: A reevaluation. Proceedings of the National Academy of Sciences of the United States of America, 94(26), 14792-14797.

Thompson-Schill, S. L., Swick, D., Farah, M. J., D’Esposito, M., Kan, I. P., \& Knight, R. T. (1998). Verb generation in patients with focal frontal lesions: A neuropsychological test of neuroimaging findings. Proceedings of the National Academy of Sciences of the United States of America, 95(26), 15855-15860.

Tzourio-Mazoyer, N., Landeau, B., Papathanassiou, D., Crivello, F., Etard, O., \& Delcroix, N. (2002). Automated anatomical labelling of activations in spm using a macroscopic anatomical parcellation of the MNI MRI single subject brain. Neurolmage, 15(1), 273-289.

van Ettinger-Veenstra, H. M., Ragnehed, M., Hällgren, M., Karlsson, T., Landtblom, A. M., Lundberg, P., et al. (2010). Right-hemispheric brain activation correlates to language performance. NeuroImage, 49(4), 3481-3488.

Vigneau, M., Beaucousin, V., Hervé, P. Y., Duffau, H., Crivello, F., Houdé, O., et al. (2006). Meta-analyzing left hemisphere language areas: Phonology, semantics, and sentence processing. NeuroImage, 30(4), 1414-1432.

Wada, J., \& Rasmussen, T. (1960). Intracarotid injection of sodium amytal for the lateralization of cerebral speech dominance: Experimental and clinical observations. Journal Neurosurgery, 17, 266-282.

Wagner, A. D., Paré-Blagoev, E. J., Clark, J., \& Poldrack, R. A. (2001). Recovering meaning: Left prefrontal cortex guides controlled semantic retrieval. Neuron, 31(2), 329-338.

Whitehouse, A. J., \& Bishop, D. V. (2008). Cerebral dominance for language function in adults with specific language impairment or autism. Brain, 131(12), 3193-3200.

Wildgruber, D., Ackermann, H., \& Grodd, W. (2001). Differential contributions of motor cortex, basal ganglia, and cerebellum to speech motor control: Effects of syllable repetition rate evaluated by fMRI. Neurolmage, 13(1), 101-109.

Xiong, J., Rao, S., Jerabek, P., Zamarripa, F., Woldorff, M., Lancaster, J., et al. (2000). Intersubject variability in cortical activations during a complex language task. NeuroImage, 12(3), 326-339. 